\title{
MUNICIPAL PUBLIC TRANSPORT LINE MODELLING
}

This contribution deals with modelling and simulation of a transport system of a public transport line as one of the ways leading to deeper insight into the system and thus also to its development and its quality and effectiveness improvement. Professional software for simulation model creation was used for implementation of the model. Questions related to accessibility of real data for the model setting and their processing will also be discussed.

Keywords: Simulation, transport system modelling, public transport.

\section{Introduction}

Transport systems represent a strategic sphere for each society or organization, their operation is vital for such a society. This is why permanent attention is particularly paid to effectiveness of these systems. Modelling and simulation of these systems is one of the ways leading to better understand them. This is what this contribution is also focused on.

There are several approaches to choose from in modelling method selection. The specific choice is usually based on both, the aim we create the model for and the set of data we have available for creation of the model.

Modelling by means of so called system dynamics [1] is the first approach. This approach (identified as SD) is based on the view of the modelled system as a whole and requires input data at the same level (i.e. global). SD approach usually strives for exact (structural) modelling, i.e. a system model based on the same structures as in the modelled system. The "black box" method may however also be used when the system is only modelled by its inputs and adequate outputs without imitating its internal structure.

Modelling upon discrete events (DE) is the second possibility. In this case the modelled subject is characterized by events taking place inside that and activities related to them. This is thus a process modelling method.

Creation of a model upon data of behaviour of its parts, objects - agents of the same type the model consists of, which are usually multiple, is the third possible way. Behaviour of individual elements actually creates behaviour of the system as a whole. This method may also be advantageously used in transport systems which consist of, e.g., means of transport, transported people etc. These are then so called multi agent models (MA) [2]. This approach is usually used where objects - agents show some signs of autonomous behaviour.

A method based on so called state diagrams, which is similar to the DE approach, but where initiation of an event is not bound to time but more likely to a met condition, may also be used for modelling. Algorithmic modelling based on flow charts as a subject behaviour description tool may also be used. In practice we may also see hierarchical models enabling separation and independent modelling of different levels of the system or its components even by means of different techniques.

The purpose of this contribution is to show application of modelling techniques (particularly DE) to modelling of a transport system consisting of one public transport line using professional software for simulation model creation. The aim is then to show that the created model can be applied to detection of problematic points in operation of such a line and to its optimization.

\section{Present state of art}

Upon research of available resources we may observe that particularly DE models are applied to transport system, but MA models have been used more and more often recently. As a whole they may also be modelled on SD bases. Particularly the multi-agent modelling based on cellular automates proves to be very interesting. Its application has particularly increased in recent years, when instruments supporting this approach have arisen. An example of MA approach to modelling of a crossroad or a more complex system (St. Petersburg Metro) can be found in [3]. It is obvious particularly on the latter example how complicated systems with numerous internal links and processes

\footnotetext{
* Jiri Jelinek

Institute of Technology and Business in Ceske Budejovice, Czech Republic

E-mail: jelinek@mail.vstecb.cz
} 
can be modelled by means of the MA approach. A DE model of a railway transport system can be explored at the same place. The user requirements on the transport system are discussed in [4].

AnyLogic professional tool [3] enabling also creation of heterogeneous models can be used for all the above approaches except others.

\section{Proposal of the model}

\subsection{Task characteristics}

As mentioned above an analysis of a chosen transport system and its operation and subsequent creation of a simulation model was the aim of the assignment. The data the operator has available on the system (public transport line) consist particularly of an operation schedule of the line (timetable of the services), which is divided into 3 time zones, namely the day zone ( 6 a.m. - 6 p.m.), night zone (10 p.m. - 4 a.m.) and low operation zone (4 a.m. -6 a.m., 6 p.m. - 10 p.m.). Three different service schedules are available for these zones. The schedule for the peak zone from 6 a.m. to 6 p.m. was used for the purpose of the model verification. The operator had also access to the data on delays that have cumulative character (the delay of each service arrival to the particular stop against the timetable is available for each stop).

The interval data on occupancy rate of the individual services are also available; however the starting points and destinations are not obvious from them so the due adjustment of the model from this point of view was very difficult.

The created model particularly concentrated on the passage of the service through the line route. The aim was to examine the delay values, however no more in the form of cumulative values but the delay values occurred at the individual sections of the line, always between two stops.

\subsection{Model description}

It is necessary to repeat at this point that the choice of model creation methodology has to be based on the aims we create the model for and also on the data available on the system. In our case these were the above described data and the generally known character of the system corresponding to the public service system.

A diagram based on discrete events was chosen for description of the modelled system, while one stop was the basic structure element of the diagram. Its model is in the picture [Fig. 1].

The shown model includes not only the stop itself and the vehicle behaviour at that point but also the passage throught the section from the previous stop. After the service arrival (output of the "wayTo" structure) a particular number of passengers who subsequently get off leaving the diagram by means of the "end" element. After that new passengers get on, while these are generated by the "source" and join the "queue" (the structures have identical names). The actions of getting on and off have no time duration and are included mainly because of complexity of the created model and possibility of its extension.

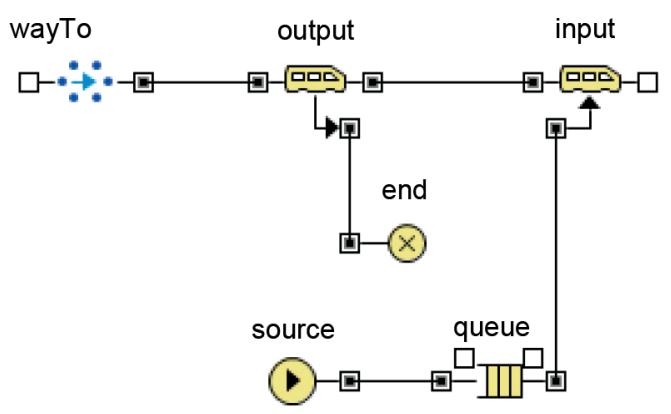

Fig. 1 DE model of a stop

The above model of the stop is repeatedly used for description of the model of the whole public transport line [Fig. 2]. There is one so called 2D histogram for each stop in [Fig. 2] showing the sum of delays the services have taken in the section immediately preceding their arrival at the particular stop. A graphic element illustrating the vehicles moving on the line was added to the diagram to make it more illustrative. A real schedule of the line was implemented in the diagram for higher credibility of the diagram.

The core of the model is particularly a mathematic description of behaviour of the services, which is mostly included in the submodel illustrating a stop. The passage time for each section $i$ between stops $i-1$ and $i$ can be determined according to formula [3]:

$$
m_{i}=\sum_{j=1}^{i} t_{j}+k_{1}-\sum_{s=1}^{i-1} m_{s}=\sum_{j=1}^{i}\left(t_{j}+e_{j}\right)-\sum_{s=1}^{i-1} m_{s},
$$

Therein $t_{i}$ is the time set by the schedule for passage of the $i$-th section and $k_{i}$ is cumulated delay at that stop. The sum of $m_{s}$ values specifies when the service really left the previous stop. Calculation of delay $e_{i}$ upon available data is substantial for simulation.

$e_{i}=\left(\sum_{j=1}^{i} t_{j}+k_{i}\right)-\left(\sum_{s=l}^{i-l} m_{s}+t_{i}\right)=k_{i}-\sum_{j=1}^{i-1}\left(m_{j}-t_{j}\right)$

The first parenthesis in the above formula [1] says when the service really arrived at stop $i$, the second when the service really left the previous stop. The expression can be transformed to another form, which already involves the cumulated delay value $k_{i}$ which was determined empirically.

After arrival at the stop (output from the "wayTo" element) the time data related to the time of passing the latest monitored section and the stay at the stop (these two steps cannot be 


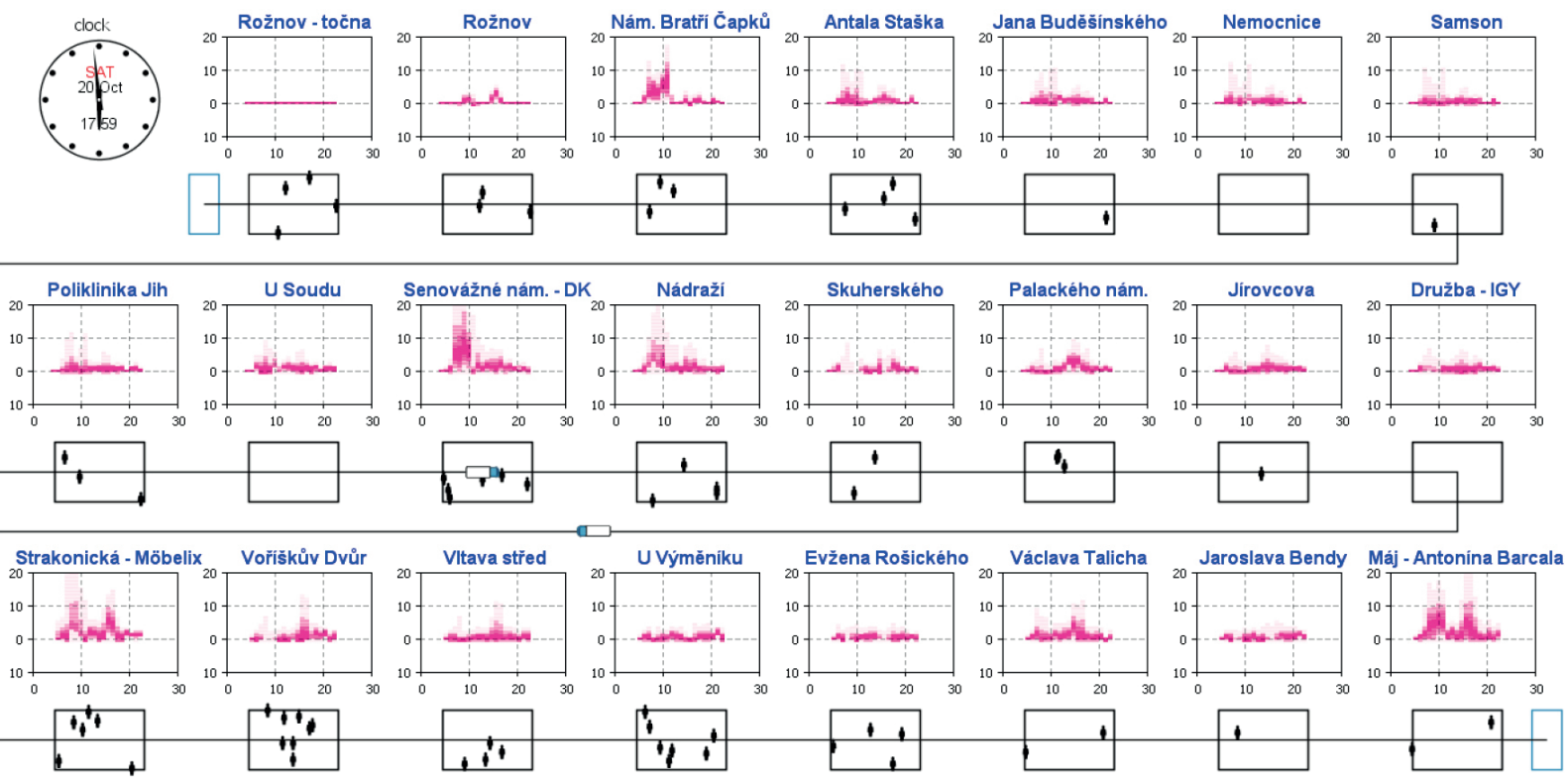

Fig. 2 The model overall structure Small graphs show the delay during the day in the section before the station

differentiated upon the available data) are stored in the data repositories. The model is even prepared for modelling the number of transported people, however the empiric data obtained from the traffic survey were not enough detailed for this purpose.

\subsection{Delay curve modelling}

The $e_{i}$ value is not in fact a constant but it is a function of the time of day (in our case time in minutes from 4:00 of each day), so $e_{i}=f_{i}(t)$. A question arose during the modelling how to model this curve for each stop. An example of the obtained values is presented in [Fig. 3] (the Delay line). Two iteration methods based on estimation of 6 parameters of approximating function $f_{i}(t)$ defined as combination of two Gauss curves (twice the mean value, standard deviation and amplitude) were tested for creation of analytic description of the presented values. Both, genetic algorithms and the Solver tool from MS Excel had been used for the estimation. The comparison shows that both the methods practically led to the same setting, just with small differences.

However application of the above approximating function leads to adjustment of constant delay values for given time without influence of stochastic events (e.g. fluctuation of traffic) and the calculation procedure is demanding. This is why random variable with triangular distribution of probability density with limits in the maximum and minimum of the chosen floating interval $\langle\mathrm{t}-15 ; \mathrm{t}+15\rangle$ (time in minutes) and with centres in the moving average was finally used for modelling delay in the model.

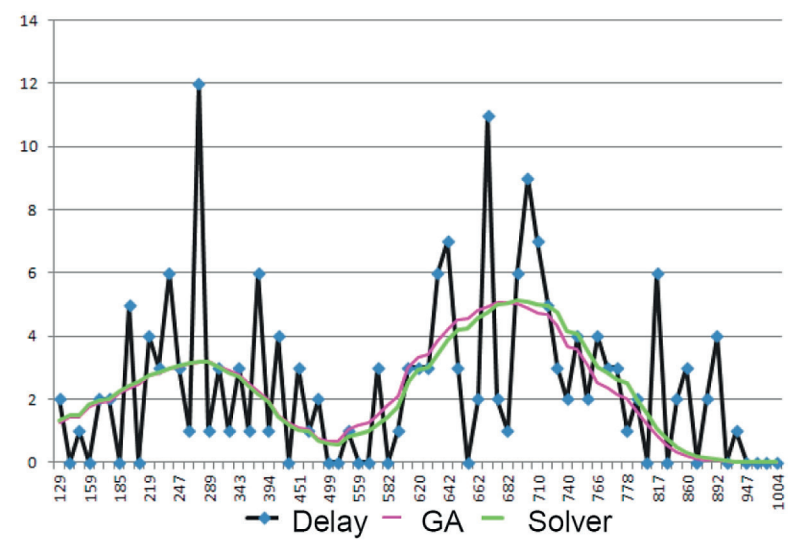

Fig. 3 Empirically determined values of cumulated delay between two stops and the course of approximation function with different parameter petting (both axes in minutes)

\section{Simulation experiments}

The proposed model was implemented in AnyLogic simulation tool. Apart from animation showing the passage of the means of transport through given line the system also shows some aggregate data. A histogram of service delay at the terminal [Fig. 4] is the first output. Delay of services in minutes goes on the $x$ axis and frequency of that delay goes on the $y$ axis.

It is obvious from the picture that the distribution is Gauss type and it clearly demonstrates that except for approximately $17 \%$ all the services are delayed. A delay of more than 10 minutes is likely for substantial percentage of services, which will influence 


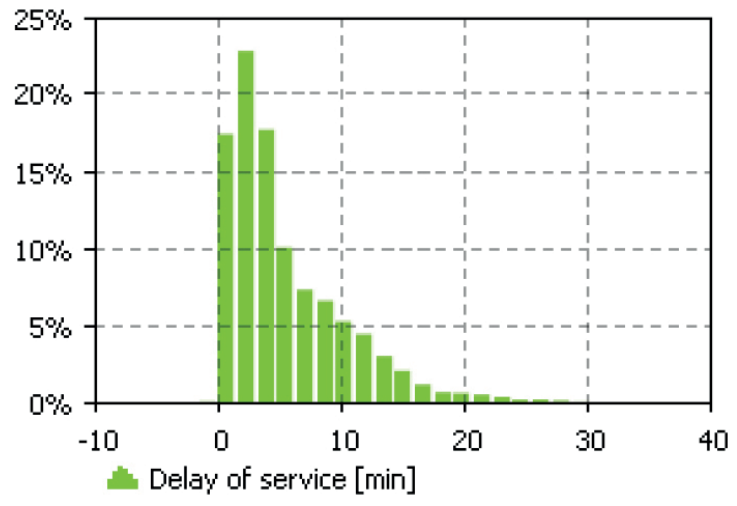

Fig. 4 Histogram of service delay

negatively the whole schedule of the line as well as linked lines (from the point of view of servicing with the same vehicles).

2D histogram of service delay distribution during a day is the second output [Fig. 5] (the more saturated colour the more delay occurrences). Time of a day in hours goes on the $x$ axis and the rate of the delay goes on the $y$ axis. It is obvious from the graph that there are two critical peaks of the delay function during the day, namely within the morning rush hour from 7 to 11 a.m. and then in the afternoon from 2 to 5 p.m. Critical moments are between 8 and 9 a.m. and between 3 and 4 p.m.

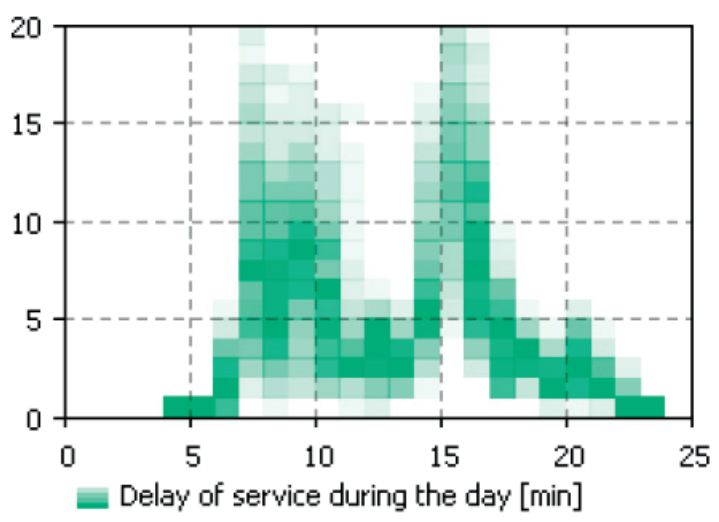

Fig. 5 2D histogram of service delay during the day (x-axis in hours, $y$-axis in minutes)

The last aggregate diagram [Fig. 6] is an estimate of waiting time for service arrival within the whole line. Time of a day in hours goes on the $x$ axis and waiting time for a service goes on the $y$ axis. This time is in fact an estimation only and is based on Poisson probability distribution $(\lambda=3)$ of times between comings of passengers to the individual stops (a simplifying premise that the numbers of passengers at the individual stops are the same was applied). The graph shows that the longest waiting times occur during the day except for morning and evening periods when intervals between the services are longer than during rush hours, particularly at about 10 a.m. - 1 p.m.; morning and afternoon rush hours are covered very well in the existing adjustment (waiting times are relatively short).

Examination of a delay occurred in a particular line section (i.e. between a chosen stop) was also interesting. An example of a histogram of this delay is in [Fig. 7]. The values show that the biggest problems occur at about 8 a.m. and 4 p.m. at this section. There should be thus more time for passage through the given section at that time. Another result is that the schedule being used, taking into account only the day, evening and night operation is insufficient. Apart from general day schedule there should also be introduced a rush-hour schedule, implementation of which might eliminate the delays in the above mentioned periods.

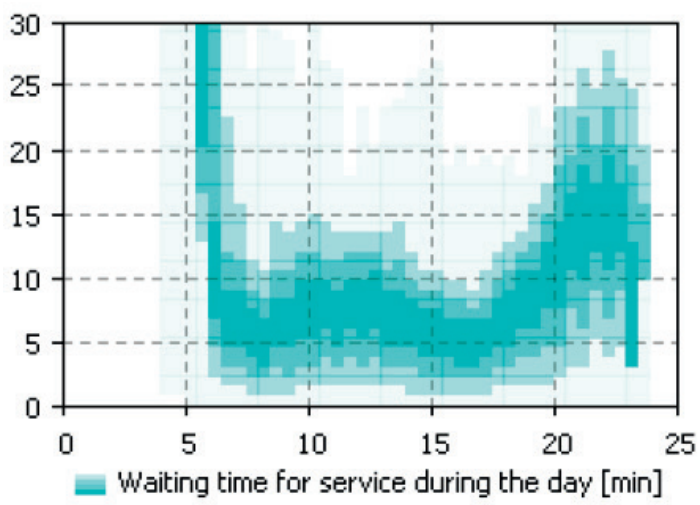

Fig. $62 D$ histogram of waiting time during the day ( $x$-axis in hours, $y$-axis in minutes)

Further simulation experiments that brought, except others, clear conclusions regarding the character and availability of input data were also performed together with the model. The presented model was based on the data from the operator; however the results have shown that data describing traffic density at the individual line sections would lead to more accurate delay simulation, however they are not available at this moment.

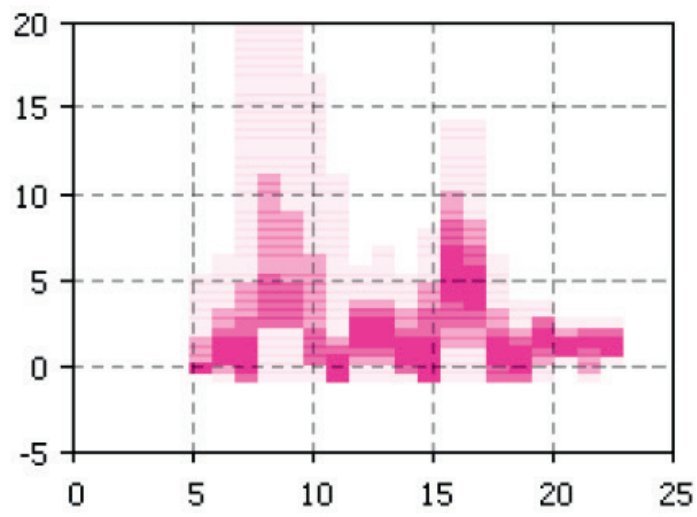

Fig. 7 - 2D histogram of delay in a particular section during the day (x-axis in hours, $y$-axis in minutes) 
Examination of numbers of transported passengers and modelling of this value upon similar empirically obtained data with suitable structure would be beneficial for further extension of the model. This would also enable us to determine more exactly the time spent by the vehicle at the bus stop, when the getting off and on would no more be an action without time dimension (like in this model). There is another result, that the time differentiation of data from the operator (e.g. data of cumulated delay) with 1 minute unit is insufficient for more exact modelling. Obtaining data directly from the board control units, complemented where necessary by further technical devices (e.g. collection of data on weight of transported people) would be an ideal solution of most of the problems.

\section{Conclusions}

The present model shows how a modelling technique based on the DE approach can be applied to a description of a public transport line, particularly from the point of view of working with time information concerning this line (time of passages of individual sections, delay rate etc.). The model may be extended by simulation of numbers of passengers of the individual services, provided these data are available.

Further work on the model will focus particularly on more exact verification of the model against the empirically obtained data. Application would be then possible, apart from the simulation itself, also to preparation of methodology of collecting data from the real system in a structure applicable also to simulation modelling and particularly extension of the scope of the proposed model (e.g. for modelling occupancy rate of services). Data from control units of vehicles might also be included.

The aim for the future is to apply the obtained experience to a design of a multi-agent model based on an active means of transport with characteristic adopted from the above described DE model. Application of such a model is particularly possible in the field of complex modelling of a public transport system (more lines).

\section{Reference}

[1] FORRESTER, J. W.: Industrial dynamics, Cambridge, MA : MIT Press, 1961

[2] SIEBERS, P. O., AICKELIN, U.: Introduction to Multi-Agent Simulation, [ed.] F. Adam and P. Humphreys. Encyclopedia of Decision Making and Decision Support Technologies, Pennsylvania : Idea Group Publishing, 2008, pp. 554-564

[3] AnyLogic Company: AnyLogic. [Online] 1992-2012, [Cited: 2. 11. 2012.] http://www.anylogic.com

[4] KAMPF, R., LIZBETIN, J., LIZBETINOVA, L.: Requirements of a Transport System User, Communications - Scientific Letters of the University of Zilina, vol. 14, No. 4, 2012, pp. 105-107. 